\title{
Specific and global processing by preschool children and college adults
}

\author{
RICHARD L. METZGER \\ Mercer University, Macon, Georgia \\ and \\ MARION PERLMUTTER \\ University of Minnesota, Minneapolis, Minnesota
}

\begin{abstract}
Possible developmental differences in global and specific processing of pictures were examined in preschool and college-age subjects. Participants were required to indicate whether comparison stimuli were the same as or different from a standard. On half of the trials, the comparison stimuli were identical to the standard, and on half they were different. The different comparison stimuli were the same background with different details, a different background with the same details, or a different background with different details. Half of the subjects were tested in a perception condition, in which the standard was present during response, and half were tested in a memory condition, in which the standard was absent during response. All subjects performed well on all item types. This finding indicates that both global and specific aspects of stimuli were perceived and remembered by young children and adults. Nevertheless, for both age groups, in both test conditions, performance was somewhat worse for samebackground/different-detail items. This finding indicates that specific information was somewhat less likely to be available or used than was global information. The patterns of reaction times for children and adults differed. The adults' data were consistent with a sequential dimension comparison model, whereas the children's data were consistent with a simultaneous dimension comparison model. The results suggest that young children and adults extract and retain similar information from complex pictures, although they use this information differently.
\end{abstract}

The present study was designed to investigate possible age differences between children's and adults' specific and global processing. In a "preception" condition, subjects' made same-different judgments between perceptually available standard and comparison pictures. In a "memory" condition, the comparison was made to a standard that was not perceptually available. Accuracy was used to assess the information subjects had available to them, and reaction time patterns indicated how the information was used.

\section{METHOD}

\section{Subjects}

Twelve males and 12 females from the University of Minnesota Institute of Child Development nursery school and 12 males and 12 females from the University of Minnesota's psychology subject pool participated in the study. Half of the subjects of each sex and age were assigned randomly to the perception condition, and the remaining subjects were assigned to the memory condition.

This research was supported by National Institutes of Health Grants PHS 1R01 HD-MH11776 and PHS 5PO1 HD-05027. Requests for reprints should be sent to Marion Perlmutter, Institute of Child Development, 51 East River Road, University of Minnesota, Minneapolis, MN 55455.

\section{Stimulus Materials}

The presentation items were modifications of stimuli used by Antes, Penland, and Metzger (1981). They were drawings of complex scenes (i.e., a kitchen, a farm), each containing four distinct objects that were appropriate for the context. The comparison stimuli were line drawings similar to the presentation stimuli. Same-background/same-detail items were identical to the standard; the correct response to them was "same." The three other types of comparison stimuli were different from the standard in background, detail, or both background and detail; the correct response to them was "different." In the samebackground/different-detail stimuli, four objects were substituted in the same locations as the objects in the original scene. In different-background/same-detail stimuli, the four objects remained the same, but the background was changed. In differentbackground/different-detail stimuli, both the background and the objects were changed.

Thirty-six standard-comparison pairs were included as test items. Half involved same items, and half involved different items, 6 of each of the three change types. Equal proportions of each item type were tested in each quarter of the test sequence. Within this constraint, items were arranged randomly, with the further restriction that a particular response was not correct for more than three consecutive trials.

\section{Apparatus}

All stimuli were presented as slides, rear-projected onto a screen containing two individually delineated presentation fields. The standard always appeared on the left field, and the comparison on the right field. Responses were made by pressing one of two buttons on a response box. 
Procedure

All subjects were tested individually, and the same test procedure was used for both the children and the adults, except that the instructions used with the children introduced the experiment as a game and the children received more extensive training to make same-different responses.

The procedures for the perception and memory conditions were basically the same, except that in the perception condition, the first picture remained present when the second picture was shown, and in the memory condition, the first picture was removed when the second picture was presented. In both conditions, the standard stimuli were shown alone for $3 \mathrm{sec}$, and then the comparison stimulus was presented. The presence of the comparison stimulus activated a timer, which measured reaction time until a same-different judgment was produced.

\section{RESULTS}

Separate 2 (age) $\times 2$ (sex) $\times 2$ (condition) $\times 2$ (background) $\times 2$ (detail) analyses of variance were performed on mean number correct and median response speed for correct responses. Follow-up simple-effects tests were carried out with the Newman-Keuls procedure. Sex of subject was not a significant factor in any of the analyses, and thus will not be discussed further.

\section{Accuracy}

The mean number of correct responses for each age group, in the perception and memory conditions, is shown for each item type in Figure 1. Overall, adults performed better than children $(22.55$ vs. 19.64$)[F(1,56)$ $=30.94, \mathrm{p}<.001]$, and performance was better in the perception than in the memory condition (22.55 vs.

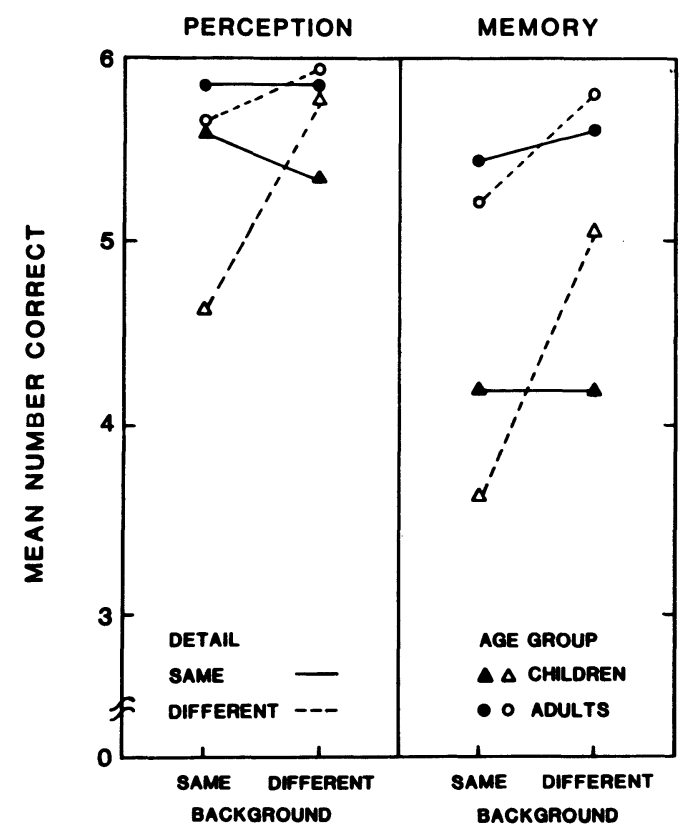

Figure 1. Mean number of correct responses in perception and memory conditions, by preschool and college students, for each item type.

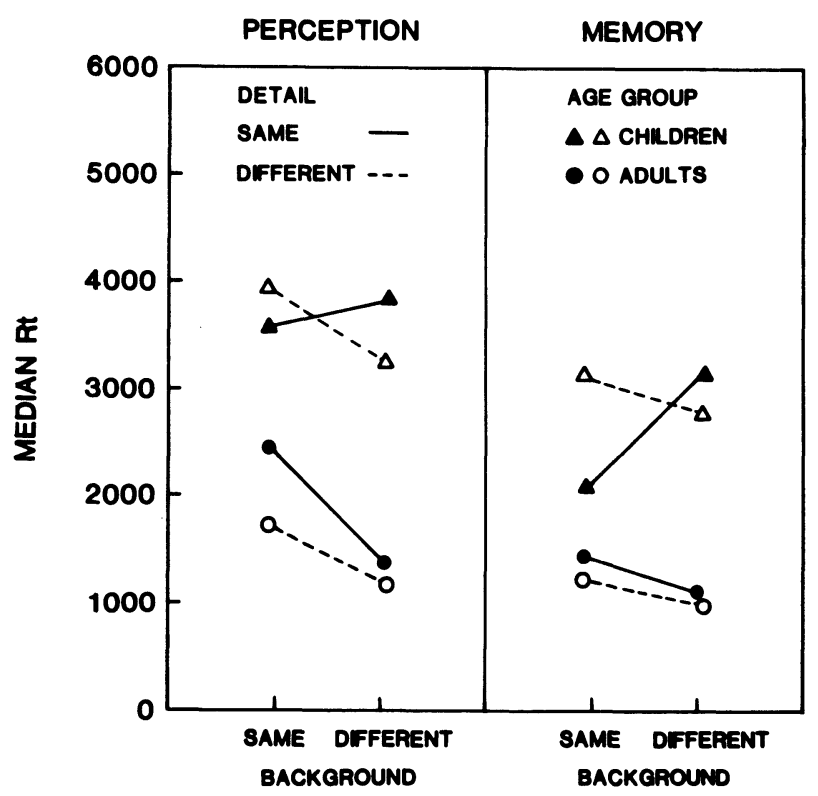

Figure 2. Median reaction times on correct responses in perception and memory conditions, by preschool and college students, for each item type.

19.94) $[\mathrm{F}(1,56)=19.45, \mathrm{p}<.001]$. There was no significant interaction between age and condition. Although there was no main effect of detail, there was a main effect of background, indicating that stimuli with changes in background were responded to more accurately than stimuli with the same background $[F(1,56)$ $=13.69, \mathrm{p}<.001]$. Moreover, there was a significant background $x$ detail interaction $[F(1,56)=12.32$, $p<.001]$. Performance was worse for same-background/ different-detail items (9.57), but not statistically different for any of the other item types $(10.75,10.67$, and 11.20). None of the interactions between these factors and age or condition were significant.

\section{Reaction Time}

The median reaction times on correct responses, for each age group, in the perception and memory conditions, are shown for each item type in Figure 2. As can be seen, adults performed faster than children $(1,375$ vs. $3,219 \mathrm{msec})[F(1,56)=144.03, p<.001]$ and performance was faster in the memory than in the perception condition $(1,979$ vs. $2,615 \mathrm{msec})[\mathrm{F}(1,56)=17.15$, $\mathrm{p}<.001]$, but there was no significant interaction between age and condition. The pattern of reaction times for background and detail changes differed for the two age groups $[F(1,56)=21.17, p<.001]$. For adults, stimuli that differed in either background or detail were responded to more rapidly than stimuli that were the same on these dimensions. Moreover, stimuli that were different in both background and detail were responded to faster than any of the other items, and stimuli that were the same in both background and 
detail were responded to slower than any of the other items. In contrast, for children, response speeds were not affected by independent changes in background or detail. The most rapid responses for children were to stimuli that were different in both background and detail, and the slowest responses were to stimuli that were the same in either background or detail, but not both.

\section{DISCUSSION}

A striking result of the present study was the high level of accuracy for both preschool children and college adults in perception and memory tasks. The number of correct same-different judgments was well above chance for each of the four types of comparison stimuli. This result indicates that both preschool and college subjects encoded global and specific aspects of the stimuli. The perception data indicate that the subjects in both age groups were able to detect the appropriate kind of information from perceptually available stimuli, and the memory data indicate that, in both age groups, this information was available in memory.

There was, however, a systematic difference in the level of accuracy on the various comparison stimuli. In both age groups, a larger number of errors was observed on same-background/ different-detail items. This pattern suggests that, for the types of scenes used in this study, both young children and young adults were somewhat more likely to note or use global than specific information. This finding is consistent with previous research (e.g., Navon, 1977; Sheingold \& Finkel, 1977) that supports configural superiority. That is, the accuracy data suggest that from early in life global features influence the perception of local elements in arrays. It is important to note, however, that the accuracy data are not informative about whether this configural superiority is due to earlier availability of global than of local infomation during encoding. It is possible, as Miller (1981) and Palmer (1978) have suggested, that both types of information become available at the same time, but that global information is more salient and therefore captures a greater role in attention and decision processes.

The adult reaction time data indicated that comparison processing was initiated on global information. However, the reaction time patterns differed for the two age groups. Apparently, there are developmental changes in decision processing. That is, although children and adults have similar information available, they seem to use this information differently.

For adults, different-background/different-detail stimuli were responded to most rapidly, and same-background/same-detail stimuli were responded to most slowly. This pattern is consistent with a sequential dimension comparison model, in which comparison is initiated for global characteristics of stimuli and then proceeds to specific details (see Figure 3). Adults apparently first analyze stimuli for possible global differences. If any are detected, a different response is made. If none are detected, the stimuli are analyzed further for possible specific differences. If any are detected, a different response is made. If none are detected, some further analysis appears to be carried out, and then a same response is made.

Such a sequential dimension comparison model is not consistent with the pattern of reaction times that was observed for children. Rather, the children's data seem better described by a simultaneous dimension comparison model (see Figure 4). Children appear to analyze both global and specific information before a response is made. They seem to analyze first for both global and specific differences. If this initial analysis does not lead to a confirmatory judgment, the simultaneous consideration of global and specific information seems to proceed, but with a switch to a search for similarities.

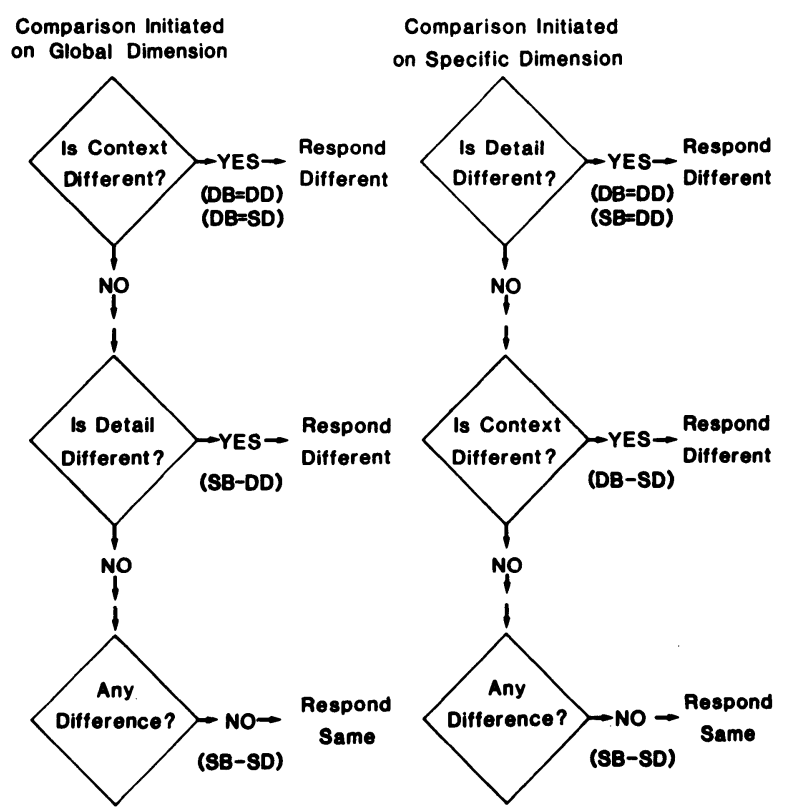

Figure 3. Representation of sequential dimension comparison modeis.

The reaction time data thus suggest that children are less efficient in their use of information than are adults. The children's judgments always were based upon both global and specific information, even when consideration of only one actually was required. This finding points to limitations in young children's understanding of the requirements of making samedifferent judgments. Similar results were obtained by Sigman and Coles (1980). In their study, young children were as adept as adults in locating criterial information in complex stimuli; however, they failed to use the information when making samedifferent judgments.

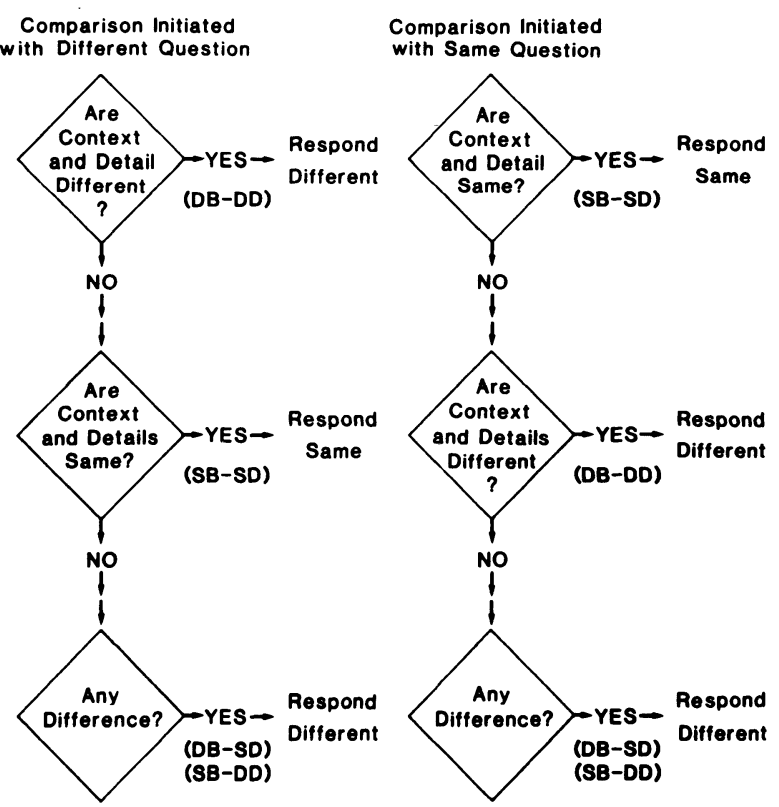

Figure 4. Representation of simultaneous dimension comparison models. 
In summary, the present data show that both global information and specific information are perceived and remembered by preschool children and college adults. The subjects made few errors in both the perception and the memory conditions. There seems, then, to be developmental stability in the way information is encoded. However, different patterns of reaction times were observed. This result suggests that there are systematic age differences in the way information is used. In particular, adults appeared more efficient in limiting their analysis to the information that was truly required for accurate performance of the same-different task.

\section{REFERENCES}

Antes, J. R., Penland, J. G., \& Metzger, R. L. (1981). Processing global information in briefly presented pictures. Psychological Research, 43, 277-292.
Mille R, J. (1981). Global precedence and decision. Journal of Experimental Psychology: Human Perception and Performance, 7, 1161-1174.

Navon, D. (1977). Forest before trees: The precedence of global features in visual perception. Cognitive Psychology, 9, 353-383.

Palmer, S. E. (1978). Structural aspects of visual similarity. Memory \& Cognition, 6, 91-97.

Sheingold, J. K., \& Finkel, D. (1977). The recognition of visual configuration and identity information in children and adults. Developmental Psychology, 13, 47-53.

Sigman, M., \& Coles, P. (1980). Visual scanning during pattern recognition in children and adults. Journal of Experimental Child Psychology, 30, 265-276.

(Manuscript received for publication February 6, 1984.) 